\title{
Identification and Analysis of Axonemal Dynein Light Chain 1 in Primary Ciliary Dyskinesia Patients
}

\author{
Judit Horváth, Manfred Fliegauf, Heike Olbrich, Andreas Kispert, Stephen M. King, Hannah Mitchison, \\ Maimoona A. Zariwala, Michael R. Knowles, Ralf Sudbrak, György Fekete, Juergen Neesen, \\ Richard Reinhardt, and Heymut Omran
}

\begin{abstract}
Department of Pediatrics and Adolescent Medicine, Albert-Ludwigs-University, Freiburg; Institut für Molekularbiologie, Medizinische Hochschule Hannover, Hannover; 'Max-Planck Institut for Molecular Genetics, Berlin; and Institute of Human Genetics, University of Goettingen, Goettingen, Germany; Department of Biochemistry, University of Connecticut Health Center, Farmington, Connecticut; Department of Medicine, University of North Carolina, Chapel Hill, North Carolina; Department of Paediatrics and Child Health, Royal Free and University College Medical School London, London, United Kingdom; and Second Department of Pediatrics, School of Medicine, Semmelweis University, Budapest, Hungary
\end{abstract}

Primary ciliary dyskinesia ( $P C D)$ is a genetically heterogeneous disorder characterized by chronic infections of the upper and lower airways, randomization of left/right body asymmetry, and reduced fertility. The phenotype results from dysfunction of motile cilia of the respiratory epithelium, at the embryonic node and of sperm flagella. Ultrastructural defects often involve outer dynein arms (ODAs), that are composed of several light (LCs), intermediate, and heavy (HCs) dynein chains. We recently showed that recessive mutations of $D N A H 5$, the human ortholog of the biflagellate Chlamydomonas ODA $\gamma$-HC, cause PCD. In Chlamydomonas, motor protein activity of the $\gamma$-ODA-HC is regulated by binding of the axonemal LC1. We report the identification of the human (DNAL1) and murine (Dnal1) orthologs of the Chlamydomonas LC1-gene. Northern blot and in situ hybridization analyses revealed specific expression in testis, embryonic node, respiratory epithelium, and ependyma, resembling the DNAH5 expression pattern. In silico protein analysis showed complete conservation of the $\mathrm{LC} 1 / \gamma-\mathrm{HC}$ binding motif in DNAL1. Protein interaction studies demonstrated binding of DNAL1 and DNAH5. Based on these findings, we considered DNAL 1 a candidate for PCD and sequenced all exons of DNAL1 in 86 patients. Mutational analysis was negative, excluding a major role of DNAL1 in the pathogenesis of PCD.

Keywords: cilia; primary ciliary dyskinesia; light chain; dynein

Congenital dysfunction of motile cilia and flagella in primary ciliary dyskinesia (PCD, MIM 242,650) (1) causes a complex phenotype (2). The epithelia of the upper and lower respiratory tract are densely covered with motile cilia, which constantly move inhaled particles, cell debris, and microbes toward the throat. Lack of mucociliary clearance due to immotile or dysmotile cilia in patients with PCD leads to recurrent respiratory infections that often cause permanent lung damage such as bronchiectasis. Dysfunction of nodal cilia during early embryogenesis causes randomization of left/right body asymmetry (3), which explains that half of the affected individuals with PCD exhibit situs inversus. The association of PCD and situs inversus is also referred to as Kartagener's syndrome (KS, MIM 244,400). Male patients with PCD often report reduced fertility, which is due to dysmotile sperm tails. Recently we identified a novel

(Received in original form October 28, 2004 and in final form March 15, 2005)

This work was supported by grants to H.O. from the German Research Foundation DFG Om 6/2 and the SFB 592 and by the Zentrum Klinische Forschung, Freiburg. Correspondence and requests for reprints should be addressed to Heymut Omran, M.D., Department of Pediatrics and Adolescent Medicine, Mathildenstrasse 1, 79106 Freiburg, Germany. E-mail: omran@kikli.ukl.uni-freiburg.de

Am J Respir Cell Mol Biol Vol 33. pp 41-47, 2005

Originally Published in Press as DOI: 10.1165/rcmb.2004-03350C on April 21, 2005

Internet address: www.atsjournals.org biological mechanism leading to hydrocephalus formation, a rare associated disease manifestation of PCD. Motile cilia of ependymal cells that line the inner ventricles and the aqueduct of Sylvius generate an "ependymal flow" of the cerebrospinal fluid (4). Disruption of ependymal flow in Mdnah5 mutant mice results in obligatory triventricular hydrocephalus due to aqueduct closure. In patients with PCD the risk for aqueduct stenosis and consecutive triventricular hydrocephalus is significantly increased.

Electron microscopy of respiratory cilia from patients with PCD often reveals characteristic ultrastructural defects (5), with partial or total absence of dynein arms as the most frequent abnormality (6). Outer (ODAs) and inner (IDAs) dynein arms contain the molecular motors that generate the force to move cilia by ATP-dependent reactions. ODAs are large multiprotein complexes that consist of heavy (HCs, 400-500 kD), intermediate (ICs, 55-110 kD), and light (LCs, 8-45 kD) dynein chains (7).

The biflagellate green alga Chlamydomonas reinhardtii has been widely used as a model organism to study assembly and function of the flagellar axoneme. Here, a number of mutants with ODA and IDA defects have been reported (8). For example, mutations of the genes encoding the ODA components IC1 and $\gamma$-HC resulted in slow-swimming algae with ODA defects (9).

Using candidate approaches and positional cloning strategies, subsequently recessive mutations in the orthologous human ODA genes DNAII and DNAH5 have been identified in patients with PCD $(10,11)$. Recently, we have shown that DNAH5 and DNAH9 mislocalization is present in respiratory epithelial cells in patients with DNAH5 and DNAI1 mutations (12). Mutations in DNAH11 encoding a dynein $\mathrm{HC}$, have been described in a patient with PCD and normal axonemal structure (13). Ciliogenesis and maintenance of cilia and flagella represents a complex mechanism that requires many individual components to be correctly assembled onto the axonemes. This is proposed to be accomplished by a cilia/flagellar-specific transport machinery. However, because generation of ciliary substructures (e.g., ODAs) is currently only at the beginning of being elucidated, characterization and mutational analysis of candidate genes has been employed to identify novel PCD genes over the past years. These studies have greatly enhanced our understanding of the pathogenic mechanisms involved in the genetically heterogenous disease PCD. Because defects of ODAs are among the most common ultrastructural abnormalities observed in PCD, we began to identify and characterize human genes encoding ODA components. Based on orthology to Chlamydomonas we recently identified the human LC-gene TCTEX (14).

Here, we report the identification and characterization of the human (DNAL1) and murine (Dnal1) ortholog of the Chlamydomonas ODA-LC gene LC1. DNAL1 and Dnal1 span $49 \mathrm{~kb}$ and $25 \mathrm{~kb}$, respectively, and comprise eight coding exons. The 
DNAL1 transcript harbors a 573-bp ORF and encodes a 190amino acid (aa) protein. We show that DNAL1/Dnal1 is specifically expressed only in tissues carrying motile cilia or flagella. We show by computational three-dimensional protein structure comparison that the $\gamma$-HC binding motif of LC1 has been completely preserved in DNAL1, and demonstrate interaction with DNAH5 by co-immunoprecipitation. Although DNAL1 represents a promising candidate gene for PCD, we did not find mutations in this gene in affected individuals of 86 families with PCD originating from Europe and the United States.

\section{MATERIALS AND METHODS}

\section{Identification of the Human DNAL1 Gene and its Mouse Ortholog Dnal1}

We identified the human ortholog of the Chlamydomonas LC1-gene by an in silico cloning strategy. The human EST database was screened with the Chlamydomonas LC1 protein sequence (AAD41040) using the BLAST program at NCBI (http://www.ncbi.nlm.nih.gov/BLAST/). We obtained a clone (AA975229), which shared high homologies with the Chlamydomonas LC1 gene and contained the human DNAL1 coding region except exon 1 . The putative exon 1 and $5^{\prime} \mathrm{UTR}$ were identified by genomic BLAST and TWOBLAST analyses using a 14q24.3 DNAL1 genomic contig derived from two PAC clones (AC005225 and AC006146) and the Chlamydomonas LC1 cDNA sequence (AF112476). The putative intron-exon structure of $D N A L 1$ was derived from genomic and cDNA sequence alignments. The preliminary exon 1 and 5'UTR of DNAL1 were confirmed by 5'-RACE using a human testis cDNA library (MarathonReady cDNA; BD Biosciences Clontech, Heidelberg, Germany) Nested amplification (Marathon cDNA Amplification Kit; Clontech) was performed following the manufacturer's instructions with the reverse primers GSP_h 5'-TCT CGC AAT TAG CAA GCA TGG AAG G-3' and nGSP_h 5'-CAT CTT CTC TAT AGG GGG AAT CTG G-3'. A 250-bp amplification product was isolated and sequenced, and the fulllength cDNA sequence was transmitted to GenBank under the Accession number AF542071. Using a similar strategy including a mouse cDNA clone (BG916281) and a BAC clone (AC125071) from mouse chromosome 12 , we also identified the murine ortholog Dnall. The $5^{\prime}$-end of the murine cDNA was amplified by $5^{\prime} \mathrm{RACE}$ using a mouse kidney cDNA library (Clontech) and the primers GSP_m 5'-TCC TCA AGT TTT TTA GGC CAT TCA GGT TGG-3' and nGSP_m 5'-ACA GAG AAA GCT TCT CGC AGT TAC C-3'.

\section{Nothern Blot}

A human multiple tissue Northern blot (Clontech) was hybridized over night at $60^{\circ} \mathrm{C}$ in Church buffer $(500 \mathrm{mM}$ phosphate buffer $\mathrm{pH} 7.2,7 \%$ SDS [wt/vol], $1 \mathrm{mM}$ EDTA supplemented with $100 \mu \mathrm{g} / \mathrm{ml} \mathrm{Salmon} \mathrm{sperm}$ DNA) using an 800-bp NotI-EcoRI fragment derived from the human cDNA clone AA975229 as a probe. The DNAL1-specific probe was radiolabeled with $\alpha-{ }^{32} \mathrm{P}-\mathrm{dCTP}$ using a Megaprime DNA labeling kit (Amersham, Freiburg, Germany). The filter was washed twice for $5 \mathrm{~min}$ at room temperature with $2 \times \mathrm{SSC} / 0.1 \% \mathrm{SDS}$ and twice for 10 $15 \mathrm{~min}$ at $55^{\circ} \mathrm{C}$ with $0.2 \times \mathrm{SSC} / 0.1 \% \mathrm{SDS}$. The membrane was exposed to an X-ray film (X-OMAT AR; Kodak, Stuttgart, Germany) in the presence of an intensifying screen (Cronex; DuPont, Bad Homburg, Germany) at $-70^{\circ} \mathrm{C}$. Equal loading of RNA was confirmed by the manufacturer of the premade blot (Clontech).

\section{In Situ Hybridization Analysis of Dnal1}

A 1.39-kb digoxigenin-labeld antisense riboprobe derived from the murine EST clone BG916281 was used for whole mount (embryonic mice postcoital day 7.5) and section (adult mice) in situ hybridization as described previously $(15,16)$. Color reactions were extended up to $4 \mathrm{~d}$ to visualize the weak expression at the node. Specimens were transferred into $80 \%$ glycerol and photographed using a Leica DC200 digital camera on a Leica M420 photomicroscope (Leica, Solms, Germany) or under Nomarski optics using a Fujix digital camera HC300Z on a Zeiss Axioplan (Zeiss, Jena, Germany).

\section{In Silico Protein Analysis}

The program ClustalW at European Bioinformatics Institute was used for alignments of the human DNAL1, mouse Dnal1, Chlamydomonas
LC1, and sequences of putative orthologous proteins from other species. Based on the published three-dimensional structure of Chlamydomonas LC1 (17), the structure of DNAL1 was calculated with use of the DALI server (www.ebi.ac.uk/dali/) and the protein data bank (www.rcsb.org/ $\mathrm{pdb} /$ ). Figures were prepared with the MOLMOL program (18).

\section{Protein Interaction Studies}

HEK293T cells were transiently transfected by calciumphosphate coprecipitation with pEGFPc2 (Clontech) as control and a pEGFPc2DNAL1 construct containing the human full-length DNAL1 cDNA fused in-frame to the C-terminal end of EGFP. Cells were harvested $24 \mathrm{~h}$ after transfection and cell lysates were prepared by NP40 lysis. Similar expression levels were confirmed by Western blot. To obtain sufficient amounts of dynein HC proteins, high-salt axonemal extracts were prepared from a pig trachea as previously described $(19,20)$. Cell lysates and protein extracts were mixed together and incubated overnight with gentle agitation at $4{ }^{\circ} \mathrm{C}$. Immunoprecipitation was performed using a Protein G Immunoprecipitation Kit (Sigma, Taufkirchen, Germany) following the manufacturer's instructions and rabbit anti-GFP antibodies (Clontech). Precipitation products were separated on NuPAGE 4-12\% Bis-Tris gels (Invitrogen, Karlsruhe, Germany) and blotted onto PVDF membranes (Amersham). Blots were processed according to standard methods using a 1:1,000 dilution of rabbit anti-DNAH5 antibodies (12) and a 1:2,500 dilution of horseradish peroxidase-conjugated anti-rabbit secondary antibodies (Santa Cruz, Heidelberg, Germany). Bands were visualized with ECL plus (Amersham).

\section{Mutational Analysis}

We analyzed affected PCD individuals from 86 families for DNAL1 mutations. We included 110 PCD families originating from different families obtained through international collaborations (Germany, Switzerland, the United States, and Hungary) in the study. Twenty-four PCD families were excluded, because DNAH5 or DNAI1 mutations, or linkage to the DNAH5 locus, was identified. The remaining 86 PCD families were included in the DNAL1 mutational analysis. We analyzed from each family one affected individual. All affected individuals were white. Forty-eight patients $(56 \%)$ had associated situs inversus. Diagnosis was based on established criteria (6). Samples from five healthy donors were included as controls. Signed and informed consent was obtained from patients and family members using protocols approved by the Institutional Ethics Review Board at the University of Freiburg and collaborating institutions. Genomic DNA was isolated by standard methods directly from blood samples or after EBV transformation of peripheral blood lymphocytes. The eight coding exons including the corresponding intron-exon boundaries of $D N A L 1$ were amplified and sequenced using following primers: Exon1F 5'-TAC TCC TGT GCG GGG TTA AG-3', Exon1R 5'-ACC CTT TTC CAC TCC CAA TC-3', Exon2F 5'-CCA AGG CTA ATA GTT ATT GCT TG-3' Exon2R 5'-TCA TGC ATC CAC CAC CAT AG-3', Exon3F 5'-CTG GCC TCT TTT GTT TCC AC-3', Exon3R 5'-TGT TTG ACC TTG CCA AAG AG-3', Exon4F 5'-ACC ATA TTG CCC TCA AGA AG-3', Exon4R 5' -CAA CAA GTA TGG CTA AAA CAG GTG-3', Exon5F 5'-CCA GCA ATT TAA GAC CAT GTG-3', Exon5R 5'-GTG GGA GTA CAG GTT GTT GC-3', Exon6F 5'-GAC AGG CAG GGT CTA AGT TCA C-3', Exon6R 5'-TTT GCA TCT TTT CCC TCT CG-3', Exon7F 5'-CAC CCG GCC AAA ACT TAG TG-3', Exon7R 5'-CTC CTC TTC ATG ATT CCA TGC-3', Exon8F 5'-ATA GTA TCT CAG AGC TTC TCA GG-3', Exon8R 5'-AGG TTG GCA TTA CCA GTT TTG-3'. PCR was performed in a volume of $50 \mu \mathrm{l}$ containing $50 \mathrm{pmol}$ primers, $2 \mathrm{mM}$ dNTPs, and $1.5 \mathrm{U}$ of Taq DNA polymerase (Invitrogen). Q-Solution (Qiagen, Hilden, Germany) was added to amplify exon 1. Amplification was performed with denaturation at $94^{\circ} \mathrm{C}$ for $4 \mathrm{~min}$ and 32 cycles of denaturation for $30 \mathrm{~s}$ at $94^{\circ} \mathrm{C}$, annealing for $30 \mathrm{~s}$ at $58^{\circ} \mathrm{C}$ for exon 1 and exon $3,61^{\circ} \mathrm{C}$ for exon $2,4,5,6,7$, and 8 , respectively, and extension for $60 \mathrm{~s}$ at $72^{\circ} \mathrm{C}$. Final extension was performed at $72^{\circ} \mathrm{C}$ for 10 min. PCR products (exon 1, $279 \mathrm{bp}$; exon 2, $614 \mathrm{bp}$; exon 3, $322 \mathrm{bp}$; exon 4, $287 \mathrm{bp}$; exon 5, $501 \mathrm{bp}$; exon 6, $314 \mathrm{bp}$; exon7, $271 \mathrm{bp}$; and exon $8,624 \mathrm{bp}$ ) were gel-extracted or column-purified (Qiagen) and directly sequenced. All exons were sequenced bidirectionally. Sequence data were compared with the corresponding genomic sequences using TWOBLAST. The quality of the sequence data was judged using Chromas (www.technelysium.com). 


\section{RESULTS}

Identification of the Human and Murine Axonemal Dynein LC1 Genes DNAL1 and Dnal1

Applying an in silico cloning strategy (see MATERIALs AND MethoDs for details), we identified the genetic structure of the human and mouse axonemal dynein LC1, DNAL1 and Dnal1. The corresponding cDNAs were confirmed by sequencing of EST clones, 5'RACE, and PCR amplification from cDNA libraries. DNAL1 spans $49 \mathrm{~kb}$, comprises eight exons, and maps to chromosome 14q24.3. All intron-exon boundaries conform to the consensus sequences of the $5^{\prime}$ and $3^{\prime}$ splice sites. The putative translation start sequence matches the Kozak consensus sequence. The mRNA harbors an 573-bp open reading frame that encodes the 190-aa DNAL1 protein. The predicted amino acid sequence is $54 \%$ identical and $69 \%$ homologous to the LC1 of Chlamydomonas rheinhardtii (Figure 1). The murine ortholog Dnal1 localizes to chromosome 12 , spans $25 \mathrm{~kb}$, and contains eight coding exons. Due to the longer 3'UTR, the transcript is $1.6 \mathrm{~kb}$ in length. The 570 -bp ORF encodes a 190-aa protein. The predicted murine protein Dnal1 is $93 \%$ identical and $97 \%$ homologous to the human DNAL1 (Figure 1). The strong homology of the human, murine, and Chlamydomonas proteins, as well as the putative orthologous proteins from other species, indicates that the function of this protein has been conserved in motile cilia and flagella from higher organisms.
High Evolutionary Conservation of the Three-Dimensional Axonemal Dynein LC1 Protein Structure

As shown in Figure 1, the LC1 and DNAL1 amino acid sequences deviate considerably within their N-terminal parts (aa 12-33), within the C-terminal half (aa 153-170 including a 3-aa deletion), and at the C-terminus (a 5-aa truncation). To test whether these sequence variations account for changes of the steric protein properties, we performed computational protein modeling. Based on the solution structure of the Chlamydomonas LC1, we predicted the three-dimensional structure of DNAL1 with an overall root-mean-square deviation (rmsd) of $0.4 \AA$ using the DALI protein structure analysis program (Figure 2). These results show that the three-dimensional structures of both orthologous proteins have been highly conserved during evolution. Only subtle differences are recognizable which do not affect the overall tertiary structures. The 3-aa deletion (aa 153-155) in the C-terminal half of DNAL1 is positioned inside the domain that forms $\alpha$-helix No. 7 of Chlamydomonas LC1. Consequently, the DNAL1 sequence is here modeled as a loop rather than an $\alpha$-helix. The C-terminal $\alpha$-helix No. 9 of the human LC is shorter by about one turn due to the 5-aa truncation. Importantly, the putative functional LC1 domains mediating protein-protein interactions have been conserved in the human orthologous protein. More precisely, DNAL1 also contains the hydrophobic patch, which is predicted to mediate the protein-protein interaction between LC1 and the $\gamma$-HC of the ODA in Chlamydomonas.

\section{H. sapiens \\ M.musculus \\ C. reinhardtii \\ X.laevis \\ D. rerio \\ D.melanogaster \\ LRR_consensus}

\section{H. sapiens \\ M.musculus \\ C. reinhardtii \\ $\mathrm{X}$.laevis \\ D. rerio \\ D.melanogaster \\ LRR_consensus}

\section{H. sapiens \\ M.musculus \\ C.reinhardtii \\ $\mathrm{x}$.laevis \\ D.rerio \\ D.melanogaster \\ LRR_consensus}

\section{H. sapiens \\ M.musculus \\ C. reinhardtii \\ $x$.laevis \\ D.rerio \\ D.melanogaster \\ LRR_consensus}
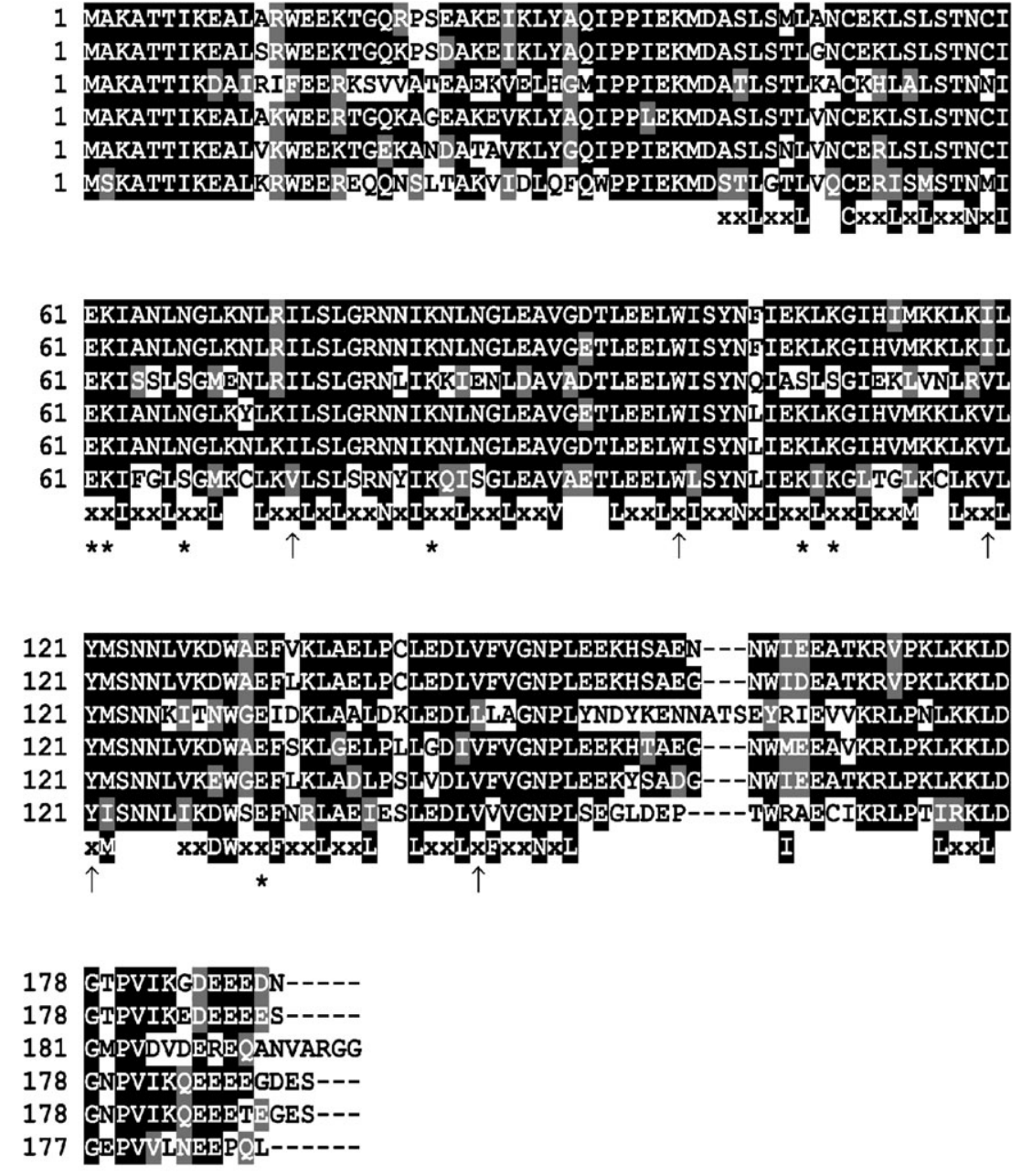

Figure 1. Sequence alignment of human DNAL1 and its murine Dnal1 and Chlamydomonas LC1 orthologs, respectively. The putative orthologs from other species were obtained by BLAST search and are also idicated. Highlighted letters and letters on a gray background represent identical and conserved amino acid residues, respectively. The LRR consensus sequence (see Discussion for details) is shown below each panel. Most parts of the orthologous proteins are highly conserved between species. The Chlamydomonas residues predicted to bind the $\gamma$-heavy chain (the ortholog of DNAH5) are marked with arrows, and the residues predicted to bind p45 are indicated with asterisks. The corresponding accession numbers are: Homo sapiens AF542071, Mus musculus BAB24259, Chlamydomonas reinhardtii AAD41040, Xenopus laevis AAH82218, Danio rerio NP_001003442, Drosophila melanogaster NP_610483. 

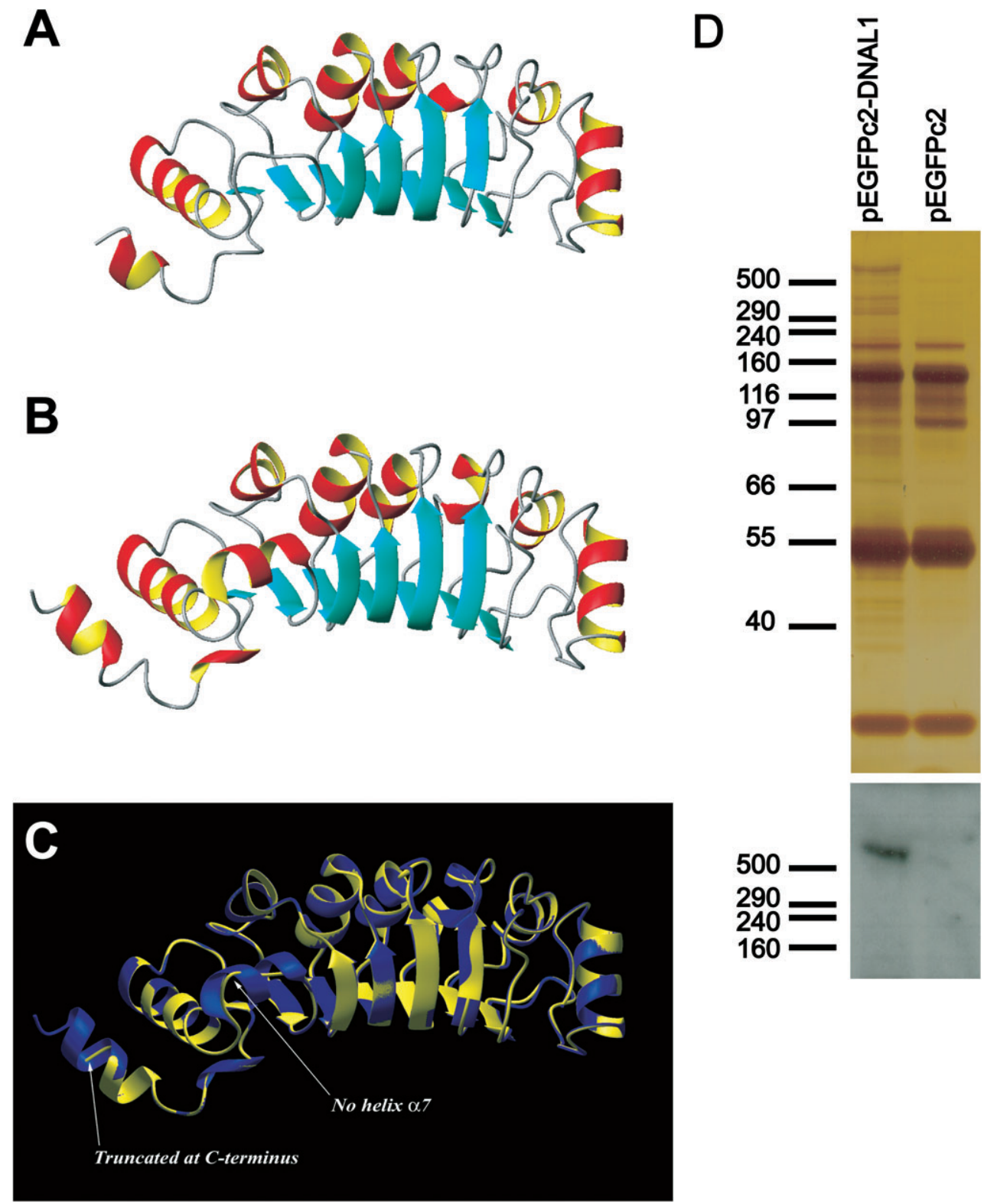

Figure 2. Alignment of the predicted three-dimensional structures of DNAL1 and the orthologous Chlamydomonas LC1. ( $A$ ) Computational modelling of DNAL1. (B) Three-dimensional structure of LC1 based on cristallographic data. (C) The overlay of DNAL1 (yellow) and LC1 (blue) elucidates the high conservation of both proteins. Minor differences are a loop instead of $\alpha$-helix No. 7 and the shortened C-terminus of DNAL1. (D) Demonstration of interaction between DNAL1 and DNAH5 by co-immunoprecipitation. Silverstaining (top) and Western blot using anti-DNAH5 antibodies (bottom) of proteins precipitated with anti-GFP antibodies from a mixtures of axonemal extracts from porcine tracheal epithelium and HEK293T cell lysates containing either an EGFP-DNAL1 fusion protein (left) or EGFP alone (right).

In addition, both proteins contain charged/polar residues on the opposite face, which represents a domain with dynamic behavior and mediates the interaction with a jet uncharacterized $45-\mathrm{kD}$ protein (17). Because of the overall structural similarities of the Chlamydomonas LC1 and the human DNAL1 (and likewise the murine Dnal1) we conclude that the protein functions, including the physical interactions, have been evolutionarily conserved.

\section{Interaction of DNAL1 with DNAH5}

To test whether DNAL1 interacts with DNAH5, the human ortholog of the Chlamydomonas $\gamma$-HC, we performed protein interaction studies in vitro. With anti-green fluorescent protein (GFP) antibodies, porcine DNAH5 could be readily co-precipitated from a mixture of an axonemal protein extract from tracheal epithelium and a lysate from HEK297T cells transiently expressing a EGFPDNAL1 fusion protein (Figure 2D). Porcine DNAH5 was not detectable by Western blot using an HEK293T cell lysate containing the EGFP alone expressed at comparable levels. From these results it can be concluded that DNAL1 and DNAH5 physically interact.

\section{Axonemal Dynein LC1 Is Specifically Expressed in Tissues Carrying Motile Cilia}

To test whether the mammalian orthologs of the Chlamydomonas $\mathrm{LC} 1$ are also associated with motile cilia function, we first analyzed the tissue specific expression of DNAL1 by hybridization of a 
multiple tissue Northern blot (Figure 3A). A specific transcript of $\sim 0.9 \mathrm{~kb}$, which correlates to the predicted transcript length, was only identified in testis. In tissues which are supposed not to carry motile cilia (spleen, thymus, colon, small intestine, peripheral blood leukocytes, prostate, and uterus) we did not observe DNAL1 expression. This result is consistent with a predicted specific role of the axonemal dynein LC1 in sperm flagella and perhaps in other motile cilia types. To confirm this hypothesis, we characterized the expression of Dnall on the cellular level by whole mount and section in situ hybridization analyses of mouse embryos and adult animals, respectively (Figures $3 \mathrm{~B}-3 \mathrm{E}$ ). In gastrulation stage embryos (7.5 d post coitum), expression was confined to the node (Figure 3B). At this developmental stage, breakage of left/right body symmetry is initiated by the activity of both motile and sensory nodal monocilia (21). Therefore the specific expression within the node during this period indicates that Dnal1 contributes to the function most likely of the motile $9+0$ cilia at the embryonic node. In sections from adult mice we found specific Dnall expression in the respiratory epithelium of the upper airways (Figure 3C) and the ependymal cells lining the brain ventricles and the aqueduct of Sylvius (Figures 3D-3E). Because both epithelial tissues are densely covered with motile $9+2$ cilia (respiratory and ependymal cilia, respectively), these results further support a putative role of the axonemal dynein LC1 in motile cilia of higher organisms.

\section{Mutational Analysis}

Although PCD represents a genetically heterogenous disease, only three PCD genes have been identified so far. These include $D N A H 5$ and DNAI1, encoding heavy and intermediate chains of the axonemal ODAs. Because of the high degree of evolutionary conservation and the putative role specifically in motile cilia, we considered the ODA LC gene DNAL1 a strong candidate gene for PCD. Therefore, we amplified and sequenced all eight coding exons of DNAL1 from a large cohort of patients with PCD comprising 86 affected individuals. Five healthy individuals were included as control subjects. However, among the patients with PCD analyzed in this study, we did not detect any sequence variants including single nucleotide polymorphisms.

\section{DISCUSSION}

Recent linkage analyses have shown that PCD represents a genetically heterogenous hereditary disease. Nevertheless, only three PCD genes have been identified to date. Whereas DNAH5 and DNAII encode heavy and intermediate chains of the ODAs, the role of DNAH11 mutations is less well understood. Because ultrastructural defects observed in PCD frequently affect the ODAs and/or IDAs, the genes encoding their components have often been proposed as candidate genes. In addition, mutations causing dysfunction of dynein arms without recognizable changes
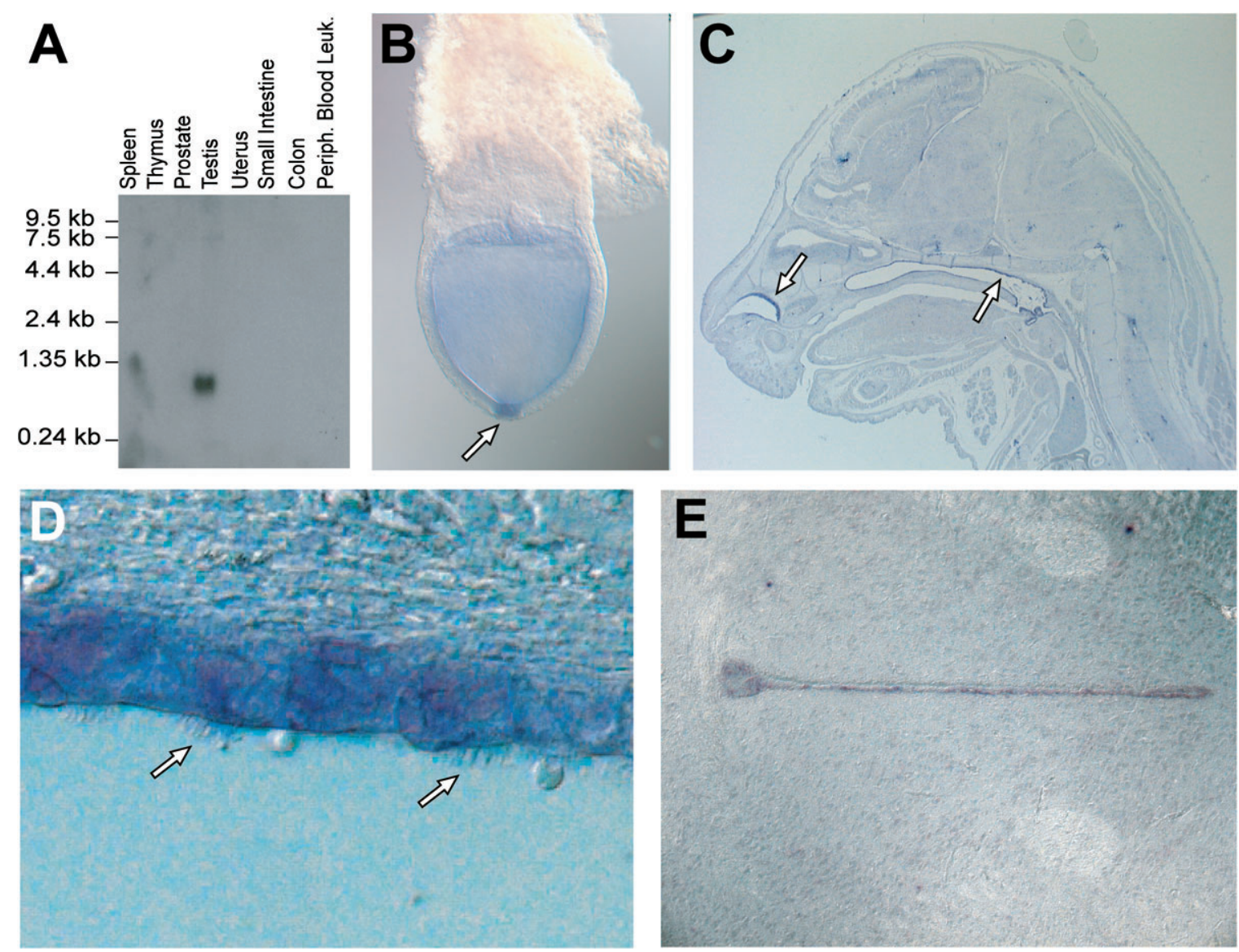

Figure 3. Expression analyses of human DNAL1 and murine Dnal1. (A) Northern blot analysis of the human DNAL1 gene shows a specific signal of the predicted size only in testis, which is compatible with expression in sperm cells. DNAL1 mRNA expression is undetectable in tissues that do not carry motile cilia. (B) Whole-mount in situ hybridization analysis of Dnal1 in early gastrulation-stage mouse embryo (7.5 d.p.c.) shows specific expression in the node (arrow). (C-E) Section in situ hybridization analysis demonstrates specific expression in ciliated respiratory epithelial cells $(C$ and $D)$ and ependymal cells of the aqueduct of Sylvius $(E)$. 
of the axonemal ultrastructures might also contribute to the genetic heterogeneity of PCD. Therefore, attempts to identify novel PCD genes have almost exclusively been based on the analysis of candidate genes. Here we describe the identification of the novel human gene DNAL1 and its murine ortholog Dnal1, encoding the axonemal dynein LC1. We applied an in silico cloning strategy, which was enabled by the evolutionary conservation of ODA proteins between the green alga Chlamydomonas and vertebrates. The high degree of amino acid sequence conservation of DNAL1, Dnal1, and the Chlamydomonas LC1 ortholog suggests that the functional role has also been evolutionarily preserved (Figure 1). This is further supported by the three-dimensional protein structures, which show only marginal differences (Figure 2).

In Chlamydomonas flagella, LC1 participates in the large multimeric ODA complex, and physically interacts with the axonemal $\gamma$-HC (22). LC1 is a member of the SDS22+ subclass of the leucin-rich repeat (LRR) family. Refined solution structure showed that the LRR region folds as a right-handed spiral with six $\beta-\beta-\alpha$-motifs that form two parallel $\beta$-sheets and an $\alpha$-helical face $(17,23,24)$. The first $\beta$-sheet exhibits a single hydrophobic patch that is predicted to bind the $\gamma-\mathrm{HC}$ in close vicinity of the ATP hydrolytic site of the motor domain. Stoichiometry measurements indicated that two LC1 molecules bind to each $\gamma$-HC. Furthermore, crosslinking experiments suggested that LC1 forms a ternary complex and also interacts with a yet unidentified $45-\mathrm{kD}$ protein (22). Therefore, it has been predicted that the motor protein activity of the $\gamma$-HC is regulated through interaction with $\mathrm{LC} 1$, which mediates the signals originating from environmental stimuli. Because of the high sequence and structural similarities, we assumed that DNAL1 might interact with DNAH5, the human ortholog of the Chlamydomonas $\gamma-\mathrm{HC}$, which we previously showed to be involved in the pathogenesis of PCD (11). Using immunoprecipitation studies we demonstrated that GFP-tagged DNAL1 binds to native DNAH5 present in axonemal extracts of pig respiratory epithelial cells (Figure 2D).

We demonstrated specific co-expression of DNAL1 (Dnal1) and DNAH5 (Mdnah5) in tissues known to carry motile cilia (respiratory epithelia and ependyma) or flagella (sperm cells) and absence of expression in non-ciliated tissues or tissues containing sensory cilia (Figure 2 ) like retina and kidney $(4,11$, $25)$. This suggests a regulatory function of DNAL1/Dnal1 for DNAH5/Mdnah5 activity in outer dynein arms of sperm flagella, respiratory cilia, and ependymal cilia.

Based on the predicted functions of DNAL1, we considered DNAL1 an excellent candidate gene for PCD. Because mutations in the Chlamydomonas $\gamma$-HC gene and human ortholog DNAH5 cause slow-swimming algae or PCD with ODA defects, respectively, we hypothesized that nonfunctional DNAL1 might also cause dysmotility of cilia and flagella due to impaired regulatory functions. However, in a large cohort of patients with PCD, we did not detect sequence variations within the coding exons of $D N A L 1$, which excludes a major role in the etiology of PCD.

One possible reason for the absence of DNAL1 mutations in patients with PCD might be an essential requirement for correct function of DNAL1 in motile cilia during development. Another possibility is that DNAL1 exhibits additional functions outside the ciliary compartment. Because cilia share a number of proteins with the cytoplasm (e.g., the retrograde intraflagellar transport process is dependent on cytoplasmic dynein motors [26]) nonfunctional components might lead to cell death rather than dysmotile cilia.

In summary, we identified the human DNAL1 and murine Dnal1 gene, respectively, elucidated the intron-exon structures and cDNA sequences, and demonstrated interaction of DNAL1 and DNAH5. The strong mRNA expression in testis suggests that DNAL1 might play a particular role in sperm flagella, and raises the possibility that $D N A L 1$ mutations are involved in male sterility.

Conflict of Interest Statement: None of the authors have a financial relationship with a commercial entity that has an interest in the subject of this manuscript.

Acknowledgments: The authors are grateful to the affected individuals and their families for their participation in this study. They thank the German patient support group "Kartagener Syndrom und Primäre Ciliäre Dyskinesie e.V." and the British PCD support group for their cooperation. They also thank Steffen Hennig and Ute Trauer for help with sequencing and Rachid Melkaoui for technical assistance.

\section{References}

1. Rott HD. Kartagener's syndrome and the syndrome of immotile cilia. Hum Genet 1979;46:249-261.

2. Ibanez-Tallon I, Heintz N, Omran H. To beat or not to beat: roles of cilia in development and disease. Hum Mol Genet 2003;12:R27-R35.

3. Nonaka S, Tanaka Y, Okada Y, Takeda S, Harada A, Kanai Y, Kido M, Hirokawa N. Randomization of left-right asymmetry due to loss of nodal cilia generating leftward flow of extraembryonic fluid in mice lacking KIF3B motor protein. Cell 1998;95:829-837.

4. Ibanez-Tallon I, Pagenstecher A, Fliegauf M, Olbrich H, Kispert A, Ketelsen UP, North A, Heintz N, Omran H. Dysfunction of axonemal dynein heavy chain Mdnah5 inhibits ependymal flow and reveals a novel mechanism for hydrocephalus formation. Hum Mol Genet 2004; 13:2133-2141.

5. Jorissen M, Willems T, Van der Schueren B, Verbeken E, De Boeck K. Ultrastructural expression of primary ciliary dyskinesia after ciliogenesis in culture. Acta Otorhinolaryngol Belg 2000;54:343-356.

6. Afzelius BA, Mossberg B, Berström SE. Immotile cilia syndrome (primary ciliary dyskinesis), including Kartagener Syndrome. In: Scriver CS, editor. The metabolic and molecular bases of inherited disease, 8th ed. New York: McGraw-Hill; 2001. pp. 3369-5238.

7. El Zein L, Omran H, Bouvagnet P. Lateralization defects and ciliary dyskinesia: lessons from algae. Trends Genet 2003;19:162-167.

8. Holzbaur EL, Vallee RB. DYNEINS: molecular structure and cellular function. Annu Rev Cell Biol 1994;10:339-372.

9. Piperno G, Huang B, Luck DJ. Two-dimensional analysis of flagellar proteins from wild-type and paralyzed mutants of Chlamydomonas reinhardtii. Proc Natl Acad Sci USA 1977;74:1600-1604.

10. Pennarun G, Escudier E, Chapelin C, Bridoux AM, Cacheux V, Roger G, Clement A, Goossens M, Amselem S, Duriez B. Loss-of-function mutations in a human gene related to Chlamydomonas reinhardtii dynein IC78 result in primary ciliary dyskinesia. Am J Hum Genet 1999;65:1508-1519.

11. Olbrich H, Haeffner K, Kispert A, Voelkel A, Volz A, Sasmaz G, Reinhardt R, Hennig S, Lehrach $\mathrm{H}$, Konietzko $\mathrm{N}$, et al. Mutations in DNAH5 cause primary ciliary dyskinesia and randomization of leftright asymmetry. Nat Genet 2002;30:143-144.

12. Fliegauf M, Olbrich H, Horvath J, Wildhaber JH, Zariwala MA, Kennedy M, Knowles MR, Omran H. Mis-localization of DNAH5 and DNAH9 in respiratory cells from primary ciliary dyskinesia patients. Am J Respir Crit Care Med 2005; 10.1164/rccm.200411-1583OC (In press)

13. Bartoloni L, Blouin JL, Pan Y, Gehrig C, Maiti AK, Scamuffa N, Rossier C, Jorissen M, Armengot M, Meeks M, et al. Mutations in the DNAH11 (axonemal heavy chain dynein type 11) gene cause one form of situs inversus totalis and most likely primary ciliary dyskinesia. Proc Natl Acad Sci USA 2002;99:10282-10286.

14. Neesen J, Drenckhahn JD, Tiede S, Burfeind P, Grzmil M, Konietzko J, Dixkens C, Kreutzberger J, Laccone F, Omran H. Identification of the human ortholog of the t-complex-encoded protein TCTE3 and evaluation as a candidate gene for primary ciliary dyskinesia. Cytogenet Genome Res 2002;98:38-44.

15. Parr BA, Shea MJ, Vassileva G, McMahon AP. Mouse Wnt genes exhibit discrete domains of expression in the early embryonic CNS and limb buds. Development 1993;119:247-261.

16. Knecht AK, Good PJ, Dawid IB, Harland RM. Dorsal-ventral patterning and differentiation of noggin-induced neural tissue in the absence of mesoderm. Development 1995;121:1927-1936.

17. Wu H, Maciejewski MW, Marintchev A, Benashski SE, Mullen GP, King SM. Solution structure of a dynein motor domain associated light chain. Nat Struct Biol 2000;7:575-579.

18. Koradi R, Billeter M, Wüthrich K. MOLMOL: a program for display and analysis of macromolecular structures. J Mol Graph 1996;14:51-55.

19. Hastie AT. Isolation of respiratory cilia. Methods Cell Biol 1995;47:93-98. 
20. Reed W, Carson JL, Moats-Staats BM, Lucier T, Hu P, Brighton L, Gambling TM, Huang CH, Leigh MW, Collier AM. Characterization of an axonemal dynein heavy chain expressed early in airway epithelial ciliogenesis. Am J Respir Cell Mol Biol 2000;23:734-741.

21. McGrath J, Somlo S, Makova S, Tian X, Brueckner M. Two populations of node monocilia initiate left-right asymmetry in the mouse. Cell 2003;114:61-73

22. Benashski SE, Patel-King RS, King SM. Light chain 1 from the Chlamydomonas outer dynein arm is a leucine-rich repeat protein associated with the motor domain of the gamma heavy chain. Biochemistry 1999; 38:7253-7264.
23. Wu H, Blackledge M, Maciejewski MW, Mullen GP, King SM. Relaxationbased structure refinement and backbone molecular dynamics of the dynein motor domain-associated light chain. Biochemistry 2003;42: $57-71$.

24. Sakato M, King SM. Desing and regulation of the $\mathrm{AAA}^{+}$microtubule motor dynein. J Struct Biol 2004;146:58-71.

25. Kispert A, Petry M, Olbrich H, Volz A, Ketelsen UP, Horvath J, Melkaoui R, Omran H, Zariwala M, Noone PG, et al. Genotypephenotype correlations in PCD patients carrying DNAH5 mutations. Thorax 2003:58:552-554.

26. Rosenbaum JL, Witman GB. Intraflagellar transport. Nat Rev Mol Cell Biol 2002:3:813-825. 